This postprint is published in:

Bulletin of the Australian Mathematical Society, Volume 94, 2016, 326-336

DOI: $10.1017 /$ S0004972716000228

\title{
On Gehring-Martin-Tan groups with an elliptic generator
}

\author{
Dušan D. Repovš and Andrei Yu. Vesnin
}

\begin{abstract}
The Gehring-Martin-Tan inequality for 2-generator subgroups of PSL $(2, \mathrm{C})$ is one of the best known discreteness conditions. A Kleinian group $G$ is called a Gehring-Martin-Tan group if the equality holds for the group $G$. We give a method for constructing Gehring-Martin-Tan groups with a generator of order four and present some examples. These groups arise as groups of finite volume hyperbolic 3-orbifolds.
\end{abstract}

Mathematics Subject Classification (2010). Primary 57M50; Secondary $20 \mathrm{H} 15$.

Keywords. Hyperbolic orbifold, discreteness condition, two-generated group.

\section{Introduction}

In this paper we are interested in discreteness conditions for groups of isometries of a hyperbolic 3 -space $\mathbb{H}^{3}$. It was shown by Jørgensen that it suffices to understand the discreteness problem for a class of two-generated groups. The most famous necessary discreteness conditions are the Shimizu lemma and the Jørgensen inequality [2]. There are some generalizations of these conditions for complex and quaternionic hyperbolic spaces, see for example $[5,9,15]$.

A description of the set of all two-generated discrete non-elementary groups of isometries $\mathbb{H}^{3}$ for which the equality holds in the Jørgensen inequality is an open problem of special interest. For many elegant results concerning this problem see $[4,8,17]$ and references therein.

We shall consider a different discreteness condition for two-generated groups which was independently proved by Gehring and Martin [8] and Tan [18]. Like the Jørgensen inequality, this condition has a form of an inequality involving the trace of one of the generators and the trace of the commutator of generators. We shall say that a group is a GMT-group if it 
can be generated by two elements for which the equality holds. The following problem arises naturally.

Problem. Find all GMT-groups.

The problem is still open, and in this paper we shall present a method to construct new examples of GMT-groups from known examples.

The most interesting example of a GMT-group is a group, related to the well-known figure-eight knot. Denote by $\mathcal{F}(n)$ the orbifold with the underlying space $S^{3}$ and the singular set the figure-eight knot $\mathcal{F}$ with singularity index $n, n \geq 4$. According to [1], orbifolds $\mathcal{F}(n)$ are extreme in the following sense: Let $L_{n}$ denote the set of all orientable hyperbolic 3 -orbifolds with nonempty singular set and with all torsion orders bounded below by $n$. Therefore

$$
L_{2} \supset L_{3} \supset L_{4} \supset \ldots \text { and } \cap L_{n}=\emptyset .
$$

Then for all $n \geq 4$, the unique lowest-volume element of $L_{n}$ is the orbifold $\mathcal{F}(n)$. A formula for vol $\mathcal{F}(n)$ was given in [21]. It was shown in [19] that $\mathcal{F}(4)$ is extreme in the sense of discreteness conditions: the orbifold group of $\mathcal{F}(4)$ is a GMT-group. Below we shall use the orbifold group of $\mathcal{F}(4)$ as a starting point for constructions of new examples of GMT-groups.

In Section 2 we shall give basic definitions and describe some properties of GMT-groups. In particular, we shall prove Lemma 2.4 which gives a method for constructing new GMT-groups. Next, we shall apply this method. In Section 3 we shall prove that some 3-orbifold hyperbolic groups related to the figure-eight knot are GMT-groups. In Section 4 we shall give examples of GMT-groups which are subgroups of the Picard group.

\section{Gehring-Martin-Tan discreteness condition}

Let $\mathbb{H}^{3}$ be the three-dimensional hyperbolic space presented by the Poincaré model in the upper halfspace. Then the boundary $\partial \mathbb{H}^{3}$ can be identified with $\overline{\mathbb{C}}$. It is well-known that the group Iso $\left(\mathbb{H}^{3}\right)$ of all orientation preserving isometries of $\mathbb{H}^{3}$ is isomorphic to

$$
\operatorname{PSL}(2, \mathbb{C})=\mathrm{SL}(2, \mathbb{C}) /\{ \pm \mathrm{I}\},
$$

where I denotes the unit matrix. In the sequel we shall not distinguish between a matrix $M \in \mathrm{SL}(2, \mathbb{C})$ and its equivalence class $\{ \pm M\} \in \operatorname{PSL}(2, \mathbb{C})$. An action of

$$
g=\left(\begin{array}{ll}
a & b \\
c & d
\end{array}\right) \in \operatorname{PSL}(2, \mathbb{C})
$$

on

$$
\mathbb{H}^{3}=\left\{(z, t) \mid z \in \mathbb{C}, t \in \mathbb{R}_{+}\right\}
$$

is defined by the following rule:

$$
g(z, t)=\left(\frac{(a z+b) \overline{(c z+d)}+a \bar{c} t^{2}}{|c z+d|^{2}+|c|^{2} t^{2}}, \frac{t}{|c z+d|^{2}+|c|^{2} t^{2}}\right) .
$$

Recall that a matrix $M \in \mathrm{SL}(2, \mathbb{C}) \backslash\{ \pm \mathrm{I}\}$ is said to be:

- elliptic if $\operatorname{tr}^{2}(M) \in[0,4)$; 
- parabolic if $\operatorname{tr}^{2}(M)=4$; and

- loxodromic if $\operatorname{tr}^{2}(M) \in \mathbb{C} \backslash[0,4]$.

In particular, a loxodromic element is said to be:

- hyperbolic if $\operatorname{tr}(M) \in(-\infty, 0) \cup(2,+\infty)$.

We shall say that an element of group $\operatorname{PSL}(2, \mathbb{C})$ is elliptic, parabolic, or loxodromic if its representative in $\operatorname{SL}(2, \mathbb{C})$ is of such type.

A group $G<\operatorname{PSL}(2, \mathbb{C})$ is said to be discrete if it is a discrete set in the matrix quotient-topology. A group $G<\operatorname{PSL}(2, \mathbb{C})$ is said to be elementary if there exists a finite $G$-orbit in $\mathbb{H}^{3} \cup \overline{\mathbb{C}}$, and non-elementary otherwise.

In 1977 Jørgensen proved [7] that a non-elementary group $G<\operatorname{PSL}(2, \mathbb{C})$ is discrete if and only if any two elements $f, g \in G$ generate a discrete group. His result motivated many other investigations of discreteness conditions for two-generated groups. In the present paper we shall discuss the necessary discreteness condition obtained in 1989 by Gehring and Martin [8] and independently by Tan [18]. We shall formulate their result as follows:

Theorem 2.1 (See $[8,18]$ ). Suppose that $f, g \in \operatorname{PSL}(2, \mathbb{C})$ generate a discrete group. If $\operatorname{tr}[f, g] \neq 1$ then the following inequality holds:

$$
\left|\operatorname{tr}^{2}(f)-2\right|+|\operatorname{tr}[f, g]-1| \geq 1 \text {. }
$$

This result makes following definitions natural. For $f, g \in \operatorname{PSL}(2, \mathbb{C})$ such that $\operatorname{tr}[f, g] \neq 1$ define

$$
\mathcal{G}(f, g)=\left|\operatorname{tr}^{2}(f)-2\right|+|\operatorname{tr}[f, g]-1| .
$$

Let $G<\operatorname{PSL}(2, \mathbb{C})$ be a two-generated group. The value

$$
\mathcal{G}(G)=\inf _{\langle f, g\rangle=G} \mathcal{G}(f, g)
$$

is referred to as the Gehring-Martin-Tan number (or, shortly GMT-number) of $G$. A two-generated discrete group $G<\operatorname{PSL}(2, \mathbb{C})$ is said to be a $G M T$ group if it can be generated by $f$ and $g$ such that $\mathcal{G}(f, g)=1$.

The following statement shows (see also [20]) that property $\mathcal{G}(f, g)=1$ implies many restrictions for $f$.

Lemma 2.2. Suppose that $f, g \in \operatorname{PSL}(2, \mathbb{C})$ generate a discrete group and $\operatorname{tr}[f, g] \neq 1$. Assume that $f$ is one of the following transformations:

(i) parabolic;

(ii) hyperbolic;

(iii) elliptic of order 2 or 3 ; or

(iv) elliptic with trace $\operatorname{tr}^{2}(f)=4 \cos ^{2}(\pi k / n)$, where $(n, k)=1, n / k \geq 6$.

Then for any $g$ we have $\mathcal{G}(f, g)>1$.

Proof. The result follows immediately from the classification of elements of $\operatorname{PSL}(2, \mathbb{C})$ and from the fact that $\mathcal{G}(f, g)$ is defined for pairs $f, g$ such that $\operatorname{tr}[f, g] \neq 1$.

(i) If $f$ is parabolic then $\operatorname{tr}^{2}(f)=4$ and therefore $\left|\operatorname{tr}^{2}(f)-2\right|=2>1$.

(ii) If $f$ is hyperbolic then $\operatorname{tr}(f) \in(-\infty, 0) \cup(2, \infty)$, so $\left|\operatorname{tr}^{2}(f)-2\right|>2$. 
(iii) If $f$ is elliptic of order 2 then $\operatorname{tr}^{2}(f)=0$, so $\left|\operatorname{tr}^{2}(f)-2\right|=2$. If $f$ is elliptic of order 3 then $\operatorname{tr}^{2}(f)=1$, so $\left|\operatorname{tr}^{2}(f)-2\right|=1$. Since $\operatorname{tr}[f, g] \neq 1$ we get $\mathcal{G}(f, g)>1$.

(iv) If $f$ is elliptic with trace $\operatorname{tr}^{2}(f)=4 \cos ^{2}(\pi k / n)$, where $(n, k)=1$ and $n / k \geq 6$, then $\operatorname{tr}^{2}(f) \geq 4 \cos ^{2}(\pi / 6)=3$, so $\left|\operatorname{tr}^{2}(f)-2\right| \geq 1$. Since $\operatorname{tr}[f, g] \neq 1$ we get $\mathcal{G}(f, g)>1$.

The following statement gives a way for finding GMT-subgroups of GMT-groups with a generator of order four.

Lemma 2.3 (See [20]). Let $\langle f, g\rangle$ be a GMT-group with $\mathcal{G}(f, g)=1$, where $f$ is elliptic of order four. Then a group generated by $f$ and $h=g f g^{-1}$ is a GMT-group with $\mathcal{G}(f, h)=1$.

The following statement gives a method for constructing GMT-groups as extensions of GMT-groups with a generator of order four.

Lemma 2.4. Let $\langle f, g\rangle$ be a GMT-group with $\mathcal{G}(f, g)=1$, where $f$ is elliptic of order four. Assume that $h \in \operatorname{PSL}(2, \mathbb{C})$ is an involution of $\langle f, g\rangle$ with one of the following conjugation actions:

(i) $h f h^{-1}=g$;

(ii) $h f h^{-1}=f^{-1}$; or

(iii) $h f h^{-1}=f g^{-1} f^{-1}$.

Then $\langle f, h\rangle$ is a GMT-group.

Proof. Since $f$ is elliptic of order four, we have $\operatorname{tr}^{2}(f)=2$, hence $\mathcal{G}(f, g)=$ $|\operatorname{tr}[f, g]-1|$ and $\mathcal{G}(f, h)=|\operatorname{tr}[f, h]-1|$. Since $h$ is an involution, it follows that either $\langle f, g\rangle$ is a subgroup of index two in $\langle f, h\rangle$, or $\langle f, g\rangle$ and $\langle f, h\rangle$ coincide. Therefore $\langle f, h\rangle$ is discrete. Recall that by [2] the identify

$$
\operatorname{tr}\left[f, h f h^{-1}\right]=(\operatorname{tr}[f, h]-2)\left(\operatorname{tr}[f, h]-\operatorname{tr}^{2}(f)+2\right)+2
$$

holds for any $f, h \in \operatorname{PSL}(2, \mathbb{C})$. Then using $\operatorname{tr}^{2}(f)=2$, we get from $(2.2)$ that

$$
\operatorname{tr}\left[f, h f h^{-1}\right]=(\operatorname{tr}[f, h]-2) \operatorname{tr}[f, h]+2 .
$$

Hence

$$
\left|\operatorname{tr}\left[f, h f h^{-1}\right]-1\right|=|\operatorname{tr}[f, h]-1|^{2} .
$$

Consider the case (i) with $h f h^{-1}=g$. Hence

$$
\left|\operatorname{tr}\left[f, h f h^{-1}\right]-1\right|=|\operatorname{tr}[f, g]-1|=\mathcal{G}(f, g)=1 .
$$

Therefore, $\mathcal{G}(f, h)=1$.

Consider the case (ii) with $h f h^{-1}=f^{-1}$. Hence

$$
\left|\operatorname{tr}\left[f, h f h^{-1}\right]-1\right|=\left|\operatorname{tr}\left[f, f^{-1}\right]-1\right|=1 .
$$

Therefore, $\mathcal{G}(f, h)=1$.

Consider the case (iii) with $h f h^{-1}=f g^{-1} f^{-1}$. Then

$$
\begin{gathered}
\left|\operatorname{tr}\left[f, h f h^{-1}\right]-1\right|=\left|\operatorname{tr}\left[f, f g^{-1} f^{-1}\right]-1\right|=\left|\operatorname{tr}\left(f g^{-1} f^{-1} g\right)-1\right| \\
=\left|\operatorname{tr}\left([f, g]^{-1}\right)-1\right|=|\operatorname{tr}[f, g]-1|=1 .
\end{gathered}
$$


Here we used that for $\alpha \in \operatorname{SL}(2, \mathbb{C})$ the relation $\operatorname{tr}\left(\alpha^{-1}\right)=\operatorname{tr} \alpha$ holds. Therefore $\mathcal{G}(f, h)=1$.

In the next section we shall realize a method given by Lemma 2.4.

\section{The figure-eight knot and related orbifolds}

Let us denote by $\mathcal{F}$ the figure-eight knot in the 3 -sphere $S^{3}$ presented by its diagram in Fig. 1. The knot group $\pi_{1}\left(S^{3} \backslash \mathcal{F}\right)$ can be easily found by the

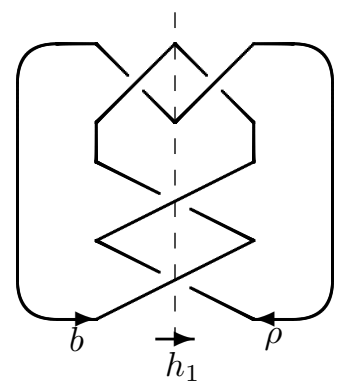

Figure 1. Generators of the group $\pi_{1}\left(S^{3} \backslash \mathcal{F}\right)$.

Wirtinger algorithm. Taking generators $b$ and $\rho$, the corresponding loops are marked in Fig. 1, we get

$$
\pi_{1}\left(S^{3} \backslash \mathcal{F}\right)=\left\langle\rho, b \mid \rho^{-1}[b, \rho]=[b, \rho] b\right\rangle,
$$

where $[b, \rho]=b \rho b^{-1} \rho^{-1}$. It is well-known [16] that the group $\pi_{1}\left(S^{3} \backslash \mathcal{F}\right)$ has a faithful representation in $\operatorname{PSL}(2, \mathbb{C})$.

Denote by $\mathcal{F}(n)$ the orbifold with the underlying space $S^{3}$ and singular set $\mathcal{F}$ with singularity index $n$, where $n \geq 4$. Cyclic $n$-fold coverings of $\mathcal{F}(n)$ are known as Fibonacci manifolds, see [13, 22] for their interesting properties. We call $\mathcal{F}(n)$ the figure-eight orbifold. Denote its orbifold group by $\Gamma_{n}=$ $\pi^{\text {orb }} \mathcal{F}(n)$. The group $\Gamma_{n}$ has the following presentation:

$$
\Gamma_{n}=\left\langle\rho_{n}, b_{n} \mid \rho_{n}^{n}=b_{n}^{n}=1, \quad \rho_{n}^{-1}\left[b_{n}, \rho_{n}\right]=\left[b_{n}, \rho_{n}\right] b_{n}\right\rangle,
$$

where generators $\rho_{n}$ and $b_{n}$ correspond to loops $\rho, b \in \pi_{1}\left(S^{3} \backslash \mathcal{F}\right)$. It is well known that for $n \geq 4$ the group $\Gamma_{n}$ has a faithful representation in $\operatorname{PSL}(2, \mathbb{C})$. According to [14], this representation is defined on generators by

$$
\rho_{n}=\left(\begin{array}{cc}
\cos \frac{\pi}{n} & i e^{d_{n} / 2} \sin \frac{\pi}{n} \\
i e^{-d_{n} / 2} \sin \frac{\pi}{n} & \cos \frac{\pi}{n}
\end{array}\right), b_{n}=\left(\begin{array}{cc}
\cos \frac{\pi}{n} & i e^{-d_{n} / 2} \sin \frac{\pi}{n} \\
i e^{d_{n} / 2} \sin \frac{\pi}{n} & \cos \frac{\pi}{n}
\end{array}\right) .
$$

The quantity $d_{n}$, defined as the complex distance between the axis of $f_{n}$ and the axis of $g_{n}$, is given by

$$
\cosh d_{n}=\frac{1}{4}\left(1+\cot ^{2}(\pi / n)-i \sqrt{3 \cot ^{4}(\pi / n)+14 \cot ^{2}(\pi / n)-5}\right) .
$$


The image of $\Gamma_{n}$ under this representation is a non-elementary discrete group. In what follows, we shall not distinguish between the group $\Gamma_{n}$ and its image under the faithful representation.

GMT-numbers of the figure-eight knot group and of the figure-eight orbifold groups were studied in [19]. It was shown that $\mathcal{G}\left(\pi_{1}\left(S^{3} \backslash \mathcal{F}\right)\right)=3$ and the following result was obtained.

Theorem 3.1 (See [19]). Let $n \geq 4$. Then the following inequalities hold for the figure-eight orbifold groups:

$$
1 \leq \mathcal{G}\left(\Gamma_{n}\right) \leq 3-4 \sin ^{2} \frac{\pi}{n}
$$

By writing the above inequalities for $n=4$ we immediately get the following:

Corollary 3.2 (See [19]). The figure-eight orbifold group $\Gamma_{4}$ is a GMT-group.

This result can be checked directly. Indeed, by (3.1) we have $\operatorname{tr}^{2}\left(\rho_{n}\right)=$ $4 \cos ^{2}(\pi / n)$, so $\left|\operatorname{tr}^{2}\left(\rho_{4}\right)-2\right|=0$. Also, by [19, Lemma 1$]$ for any $\lambda \in \mathbb{R}$ we have

$$
\left|\operatorname{tr}\left[\rho_{n}, b_{n}\right]-\lambda\right|=\sqrt{\left(\lambda^{2}-3 \lambda+3\right)+4(\lambda-1) \sin ^{2}(\pi / n)},
$$

and this gives $\left|\operatorname{tr}\left[\rho_{n}, b_{n}\right]-1\right|=1$ for any $n$. Hence $\mathcal{G}\left(\rho_{n}, b_{n}\right)=1$.

\section{Quotient orbifolds of the figure-eight orbifold}

Lemma 4.1. The figure-eight orbifold group $\Gamma_{n}, n \geq 4$, has involutions of types (i), (ii), and (iii) from Lemma 2.4 .

Proof. Being one of the simplest knots, the figure-eight knot has been intensively studied. In 1914 Dehn demonstrated [6] that the figure-eight group $\Gamma$ has eight outer automorphisms forming the dihedral group

$$
\left\langle\sigma, \tau \mid \sigma^{2}=\tau^{4}=(\sigma \tau)^{2}=1\right\rangle,
$$

where

$$
\left\{\begin{array} { l } 
{ \sigma ( \rho ) = b , } \\
{ \sigma ( b ) = \rho , }
\end{array} \quad \text { and } \quad \left\{\begin{array}{l}
\tau(\rho)=\rho b \rho^{-1}, \\
\tau(b)=b^{-1} \rho b .
\end{array}\right.\right.
$$

Later, in 1931, Magnus proved [11] that $\Gamma$ has no other outer automorphisms. It is obvious that the actions

$$
\left\{\begin{array} { l } 
{ \sigma ( \rho _ { n } ) = b _ { n } , } \\
{ \sigma ( b _ { n } ) = \rho _ { n } , }
\end{array} \quad \text { and } \quad \left\{\begin{array}{l}
\tau\left(\rho_{n}\right)=\rho_{n} b_{n} \rho_{n}^{-1}, \\
\tau\left(b_{n}\right)=b_{n}^{-1} \rho_{n} b_{n} .
\end{array}\right.\right.
$$

are outer automorphisms of $\Gamma_{n}$ for any $n$. The action of the group $\langle\sigma, \tau\rangle$ by automorphisms on $\Gamma_{n}$ is presented in Table 1 (see also [23]).

Since $\mathcal{F}(n), n \geq 4$, is a hyperbolic 3 -orbifold of finite volume (see for example [3]). Note that there are two involutions acting as described in the statement of Lemma 2.4. For any $n$ there exists $h_{1} \in \operatorname{Iso}\left(\mathbb{H}^{3}\right)$ such that

$$
\sigma\left(\rho_{n}\right)=h_{1} \rho_{n} h_{1}^{-1}=b_{n}
$$


TABLE 1. Automorphism of $\Gamma_{n}$.

\begin{tabular}{c||c|c|c|c|c|c|c} 
& $\sigma$ & $\tau$ & $\tau^{2}$ & $\tau^{3}$ & $\sigma \tau$ & $\sigma \tau^{2}$ & $\sigma \tau^{3}$ \\
\hline \hline$\rho_{n}$ & $b_{n}$ & $\rho_{n} b_{n} \rho_{n}^{-1}$ & $b_{n}^{-1}$ & $b_{n}^{-1} \rho_{n}^{-1} b_{n}$ & $b_{n}^{-1} \rho_{n} b_{n}$ & $\rho_{n}^{-1}$ & $\rho_{n} b_{n}^{-1} \rho_{n}^{-1}$ \\
\hline$b_{n}$ & $\rho_{n}$ & $b_{n}^{-1} \rho_{n} b_{n}$ & $\rho_{n}^{-1}$ & $\rho_{n} b_{n}^{-1} \rho_{n}^{-1}$ & $\rho_{n} b_{n} \rho_{n}^{-1}$ & $b_{n}^{-1}$ & $b_{n}^{-1} \rho_{n}^{-1} b_{n}$
\end{tabular}

and $\sigma$ is of type (i) from Lemma 2.4. For any $n$ there exists $h_{2} \in \operatorname{Iso}\left(\mathbb{H}^{3}\right)$ such that

$$
\sigma \tau^{2}\left(\rho_{n}\right)=h_{2} \rho_{n} h_{2}^{-1}=\rho_{n}^{-1}
$$

and involution $\sigma \tau^{2}$ is of type (ii). For any $n$ there exists $h_{3} \in \operatorname{Iso}\left(\mathbb{H}^{3}\right)$ which realizes $\tau^{2}$ with conjugation by $b_{n}$ :

$$
b_{n}\left(\tau^{2}\left(b_{n}\right)\right) b_{n}^{-1}=h_{3} b_{n} h_{3}^{-1}=b_{n} \rho_{n}^{-1} b_{n}^{-1}
$$

and involution $h_{3}$ is of type (iii).

Let us define two orbifolds with a 3 -sphere $S^{3}$ as the underlying space. Denote by $\mathcal{O}_{1}(n)$ the orbifold with singular set the spatial theta-graph presented in Fig. 2 with singularities 2, 2 and $n$ at its edges, as indicated in the figure. Denote by $\mathcal{O}_{2}(n)$ the orbifold with singular set the 2-component link $6_{2}^{2}$ with singularities 2 and $n$ at its components, as presented in Fig. 2.
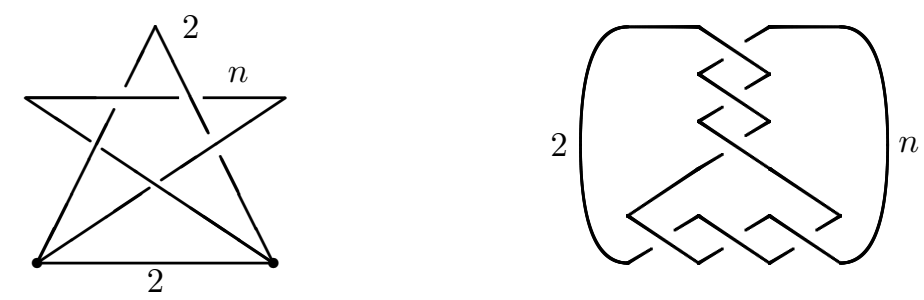

Figure 2. Singular sets of $\mathcal{O}_{1}(n)$ and $\mathcal{O}_{2}(n)$.

Theorem 4.2. The orbifold group $\pi^{\mathrm{orb}} \mathcal{O}_{1}(4)$ is a GMT-group.

Proof. For a fixed $n$ consider an involution $h_{1} \in \operatorname{Iso}\left(\mathbb{H}^{3}\right)$ from the proof of Lemma 4.1 such that $h_{1} \rho_{n} h_{1}^{-1}=b_{n}$ and $h_{1} b_{n} h_{1}^{-1}=\rho_{n}$. For $\rho_{n}$ and $b_{n}$ given by (3.1) we have $h_{1}=\left(\begin{array}{ll}0 & i \\ i & 0\end{array}\right)$. An extension of $\Gamma_{n}$ by $h_{1}$ has the following presentation:

$\Delta_{n}=\left\langle\rho_{n}, b_{n}, h_{1} \mid \rho_{n}^{n}=b_{n}^{n}=h_{1}^{2}=1, \rho_{n}^{-1}\left[b_{n}, \rho_{n}\right]=\left[b_{n}, \rho_{n}\right] b_{n}, h_{1} \rho_{n} h_{1}^{-1}=b_{n}\right\rangle$.

It is easy to see that the conjugation by $h_{1}$ is induced by an involution of $S^{3}$ whose axis corresponds to the dotted line in Fig. 1 and intersects the figure-eight knot $\mathcal{F}$ in two points. This symmetry induces an isometry, also denoted by $h_{1}$, of the orbifold $\mathcal{F}(n)$. 


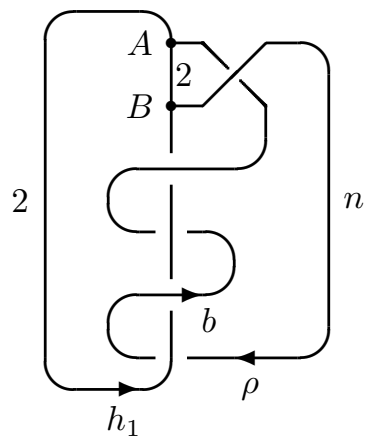

Figure 3. Singular set of $\mathcal{F}(n) / h_{1}$.

The quotient space $\mathcal{F}(n) / h_{1}$ has $S^{3}$ as its underlying space. Its singular set is a spatial graph with two vertices presented by a diagram in Fig. 3. This graph can be described as the torus knot $5_{1}$ with a tunnel $A B$. Points $A$ and $B$ are the images of intersection points of the singular set of $\mathcal{F}(n)$ with the axis of the involution $h_{1}$. Two edges of this graph, which are images of the axis of $h_{1}$, have singularity index 2 , and the third edge, which is the image of the singular set of $\mathcal{F}(n)$, has singularity index $n$.

It can be checked directly (see for example, [23] ) that the orbifold group of $\mathcal{F}(n) / h_{1}$ is isomorphic to $\Delta_{n}$ with generators $\rho, b$ and $h_{1}$, as pictured in Figure 3. Indeed, the relations (4.1) hold by the Wirtinger algorithm, in particular, the relation $\rho_{n}^{-1}\left[b_{n}, \rho_{n}\right]=\left[b_{n}, \rho_{n}\right] b_{n}$ is a consequence of the fact that the loop around its unknotting tunnel $A B$ (see [12] about unknotting tunnels) is an element of order two in the orbifold group. Obviously, spatial theta-graphs presented in diagrams in Figure 2 (left) and Figure 3 are equivalent, so $\pi^{\text {orb }} \mathcal{O}_{1}(n)=\Delta(n)$. Eliminating $b_{n}$ from (4.1) we get that $\Delta_{n}$ is a two-generated group with generators $\rho_{n}$ and $h_{1}$.

Suppose that $n=4$. By Corollary 3.2, $\Gamma_{4}$ is a GMT-group and the pair $\Gamma_{4}$ and $h_{1}$ satisfies the case (i) of Lemma 2.4. Hence $\pi^{\text {orb }} \mathcal{O}_{1}(4)$ is a GMT-group.

Theorem 4.3. The orbifold group $\pi^{\mathrm{orb}} \mathcal{O}_{2}(4)$ is a GMT-group.

Proof. To see the symmetry $h_{3}$ we shall redraw the singular set of the orbifold $\mathcal{F}(n)$ as in Figure 5. Denote $\lambda=b \rho b^{-1}$, see Figure 5. It is easy to see that $h_{3}$ corresponds to such rotational symmetry of order two that $b$ goes to $\lambda^{-1}$ and $\lambda$ goes to $b^{-1}$. Therefore, $h_{3} b h_{3}^{-1}=b \rho^{-1} b^{-1}$ that corresponds to the case (iii) of Lemma 2.4. Using $\rho=b^{-1} h_{3} b^{-1} h_{3} b$ from the defining relation $\rho^{-1}[b, \rho]=[b, \rho] b$ we get the relation

$$
b h_{3} b h_{3} b^{-1} h_{3} b^{-1} h_{3} b h_{3}=h_{3} b h_{3} b^{-1} h_{3} b^{-1} h_{3} b h_{3} b
$$

that corresponds to the canonical defining relation of the two-generated fundamental group of the two-bridge link 10/3 pictured in Figure 2 on the right (see also [23]) . Thus, the group generated by $\rho_{n}, b_{n}$ and $h_{3}$ is the orbifold 


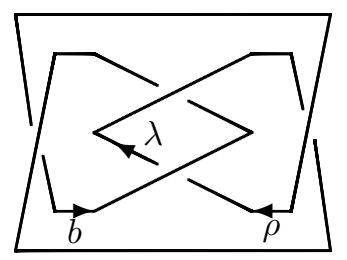

FiguRE 4. Singular set of the orbifold $\mathcal{F}(n)$.

group $\pi^{\text {orb }} \mathcal{O}_{2}(4)$. Suppose that $n=4$. By Corollary 3.2, $\Gamma_{4}$ is a GMT-group and the pair $\Gamma_{4}$ and $h_{3}$ satisfies the case (iii) of Lemma 2.4. Hence $\pi^{\text {orb }} \mathcal{O}_{2}(n)$ is a GMT-group.

\section{More examples of GMT-groups}

In this section we shall give two more examples of GMT-groups. Let us denote the orbifolds with the singular set presented in Fig. 5 by $\mathcal{O}_{3}$ and $\mathcal{O}_{4}$. The singular set of $\mathcal{O}_{3}$ is a spatial graph with two vertices that can be described as a Hopf link with an unknotting tunnel. The singular set of $\mathcal{O}_{4}$ can be described as a double link with an unknotting tunnel. Singularity indices are presented in Fig. 5. Both singular vertices of $\mathcal{O}_{3}$ belong to $\partial \mathbb{H}^{3}$. One of
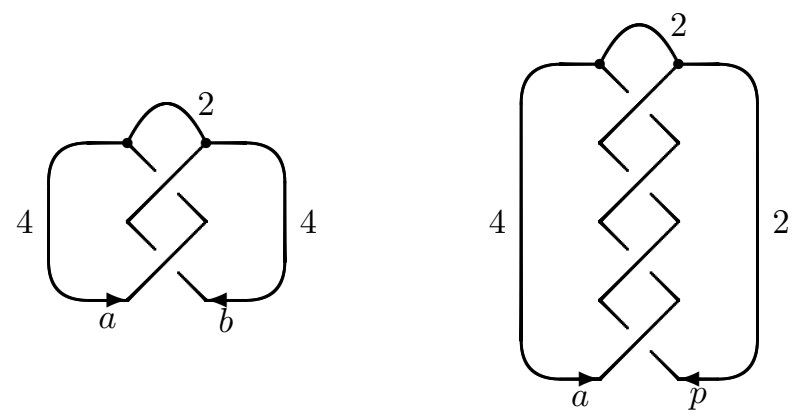

Figure 5. Singular sets of orbifols $\mathcal{O}_{3}$ and $\mathcal{O}_{4}$.

singular vertices of $\mathcal{O}_{4}$ belongs to $\partial \mathbb{H}^{3}$, whereas the other one lies in $\mathbb{H}^{3}$.

Theorem 5.1. Orbifold groups $\pi^{\mathrm{orb}} \mathrm{O}_{3}$ and $\pi^{\mathrm{orb}} \mathcal{O}_{4}$ are GMT-groups.

Proof. The orbifold group of $\pi^{\text {orb }} \mathcal{O}_{3}$ has the following presentation:

$$
\pi^{\mathrm{orb}} \mathcal{O}_{3}=\left\langle a, b \mid a^{4}=b^{4}=1, \quad[a, b]^{2}=1\right\rangle,
$$

where a relation $[a, b]^{2}=1$ corresponds to a loop around a tunnel. Concerning the hyperbolicity of this orbifold see for example, [10]. Let us use letters $a$ and $b$ also for images of generators in the group $\operatorname{Iso}\left(\mathbb{H}^{3}\right)$, corresponding to a 
faithful representation. Then $\operatorname{tr}^{2}(a)=2$ and $\operatorname{tr}[a, b]=0$. Hence $\pi^{\text {orb }} \mathcal{O}_{3}$ is a GMT-group.

It can be seen from Fig. 5 that the singular set of $\mathcal{O}_{3}$ has a symmetry of order two that exchanges $a$ and $b$. This symmetry induces an involution $\tau$ of $\pi^{\mathrm{orb}} \mathcal{O}_{3}$ defined by

$$
\tau(a)=p a p^{-1}=b \quad \text { and } \quad \tau(b)=p b p^{-1}=a
$$

for some $p \in \operatorname{Iso}\left(\mathbb{H}^{3}\right)$. It is easy to verify that the quotient orbifold $\mathcal{O}_{3} / p$ is isometric to $\mathcal{O}_{4}$. Moreover, $\pi^{\text {orb }} \mathcal{O}_{4}$ is two-generated with generators $a, p$, and $p$ satisfies the case (i) of Lemma 2.4. Hence, $\pi^{\text {orb }} \mathcal{O}_{4}$ is a GMT-group. It has the following presentation:

$$
\left.\pi^{\text {orb }} \mathcal{O}_{4}=\langle a, p| a^{4}=p^{2}=1, \quad\left(\text { apapa }^{-1} p a^{-1} p\right)^{2}=1\right\rangle .
$$

\section{Acknowledgment}

This research was supported by the Slovenian Research Agency grant BIRU/14-15-001, P1-0292, J1-6721 and J1-7025, the Ministry of Education and Science of the Russian Federation grant 16-01-00414, and the Laboratory of Quantum Topology, Chelyabinsk State University, grant 14.Z50.31.0020.

\section{References}

[1] C.K. Atkinson, D. Futer, The lowest volume 3-orbifolds with high torsion. Trans. Amer. Math. Soc., DOI: 10.1090/tran/6920, to appear. arXiv:1507.07894 [math.GT].

[2] A.F. Beardon, The Geometry of Discrete Groups. New York - Heidelberg Berlin, Springer-Verlag, 1983.

[3] M. Boileau, S. Maillot, J. Porti, Three-dimensional orbifolds and their geometric structures. Panoramas et Synthéses, 15, Société Mathématique de France, Paris, 2003.

[4] J. Callahan, Jørgensen number and arithmeticity. Conform. Geom. Dyn. 13 (2009), 160-186.

[5] W. Cao, H. Tan, Jørgensen inequality for quaternionic hyperbolic space with elliptic elements. Bull. Austral. Math. Soc. 81 (2010), 121-131.

[6] M. Dehn, Dei beiden Kleeblattschlingen. Math. Ann. 75 (1914), 402-413.

[7] T. Jørgensen, A note on subgroups of $\mathrm{SL}(2, \mathbb{C})$. Quart. J. Math. Oxford Ser. (2) 28 (1977), no. 110, 209-211.

[8] F.W. Gehring, G.J. Martin, Stability and extremality in Jørgensen's inequality. Complex Variables Theory Appl. 12 (1989), 277-282.

[9] K. Gongopadhyay, A. Mukherjee, Extremality of quaternionic Jørgensen inequality. arXiv:1503.08802 [math.CV].

[10] E. Klimenko, N. Kopteva, Two-generated Kleinian orbifolds. arXiv:math/0606066.

[11] W. Magnus, Untersuchungen fiber einige unendliche diskontinuierliche Gruppen. Math. Ann. 105 (1931) 52-74

[12] K. Marimoto, M. Sakuma, Y. Yokota, Identifying tunnel number one knots. J. Math. Soc. Japan 48 (1996), no. 4, 667-688. 
[13] S. Matveev, C. Petronio, A. Vesnin, Two-sided asymptotic bounds for the complexity of some closed hyperbolic three-manifolds. J. Austral. Math. Soc. 86 (2009), 205-219.

[14] A. Mednykh, A. Rasskazov, On the structure of the canonical fundamental set for the 2-bridge link orbifolds. Univ. Bielefeld, 1998. (Preprint 98-062, available online www.mathematik.uni-bielefeld.de/sfb343)

[15] J.R. Parker, Shimizu's lemma for complex hyperbolic space. Int. J. Math. 3 (1992), 291-308.

[16] R. Riley, A quadratic parabolic group. Math. Proc. Camb. Phil. Soc. 77 (1975), $281-288$.

[17] H. Sato, The Jørgensen number of the Whitehead link group. Bol. Soc. Mat. Mexicana 10 (2004), 495-502.

[18] D. Tan, On two-generator discrete groups of Mobius transformations. Proc. Amer. Math. Soc. 106 (1989), 763-770.

[19] A. Vesnin, A. Masley, On Jørgensen numbers and their analogues for groups of figure-eight orbifolds. Siberian Math. J. 55 (2014), 807-816.

[20] A. Vesnin, A. Masley, Two-generated subgroups of $\operatorname{PSL}(2, \mathbb{C})$ which are extreme for Jørgensen inequality and its analogues. In: Proc. Seminar on vector and tensor analysis with their applications to geometry, mechanics and physics. Moscow State University, 30 (2015), 1-54.

[21] A. Vesnin, A. Mednykh, Hyperbolic volumes of the Fibonacci manifolds. Siberian Math. J. 36 (1995), 235-245.

[22] A. Vesnin, A. Mednykh, Fibonacci manifolds as two-fold coverings over the three-dimensional sphere and the Meyerhoff-Neumann conjecture. Siberian Math. J. 37 (1996), 461-467.

[23] A. Vesnin, A. Rasskazov, Isometries of hyperbolic Fibonacci manifolds. Siberian Math. J. 40 (1999), 9-22.

Dušan D. Repovš

Faculty of Education and Faculty of Mathematics and Physics

University of Ljubljana, Slovenia

\& Institute of Mathematics, Physics and Mechanics, Ljubljana, Slovenia

e-mail: dusan.repovs@guest.arnes.si

Andrei Yu. Vesnin

Laboratory of Quantum Topology, Chelyabinsk State University, Russia

\& Sobolev Institute of Mathematics, Novosibirsk, Russia

e-mail: vesnin@math.nsc.ru 